\title{
Research on demand response strategy for promoting new energy grid connected consumption
}

\author{
Jingkai Zhu', a, Xiaotian Wang ${ }^{2, b}$ and Genzhu Lijic \\ ${ }^{1}$ State Grid Shanghai Municipal Electric Power Company, China \\ ${ }^{2}$ State Grid Shanghai Municipal Electric Power Company, China \\ ${ }^{3}$ North China Electric Power University, China \\ a1163600676@qq.com , bxtwang1982@163.com , 'lgz6869532@163.com
}

\begin{abstract}
Keywords: new energy, grid-connected consumption, demand response, power system
Abstract. New energy has developed rapidly under the current background of coping with energy crisis and environmental changes. However, new energy generation such as wind power, photovoltaic, etc. is characterized by randomness and intermittency, and new large-scale new energy incorporation brings new power to the operation of the power system. The challenge of gridlock is increasingly prominent. This article focuses on the demand response strategy to promote the integration of new energy sources and networks. First, it analyzes the role of demand response in the consumption of new energy and integrates them. Based on this, it proposes a demand response strategy that promotes new energy consumption and is based on market players. Proposed method of implementation. It aims to strengthen the stable operation of the large-scale new energy and online store systems, and improve the integration of new energy sources.
\end{abstract}

\section{Introduction}

With the increasingly severe forms of energy supply and climate change, the status of clean, low-carbon and sustainable new energy in the energy strategies of various countries has been continuously improved. The large-scale use of new energy has become a national adjustment of energy structure, guarantee of energy security, and strengthening One of the important measures for environmental protection and sustainable development ${ }^{[1]}$.

According to China's "Mid-term and long-term development plan for renewable energy," China's renewable energy consumption will reach $15 \%$ of total energy consumption by $2020^{[2]}$.At present, although the scale of new energy continues to expand, its development is facing More new challenges. The difficulty of new energy grid-connected consumption is that it has different characteristics from conventional energy sources, including randomness, volatility, and uncertainty, and brings new challenges to the grid's scheduling and operation control ${ }^{[3]}$. Therefore, there is a need to improve existing strategies, explore ways of new energy consumption, and adapt to large-scale new energy access requirements.

Demand response is essentially the use of electricity prices or incentives to encourage users to change the traditional way of using electricity, and actively participate in the energy interaction of the grid operation ${ }^{[4]}$. The demand response has changed the fixed thinking that used to rely solely on the development of the power supply side to meet the growing power demand ${ }^{[5]}$. As the demand response continues to advance, more and more demand response resources have been explored. The realization of more flexible load requirements provides a new idea for effective resolution of large-scale new energy grid-connected consumption.

\section{Analysis of the role of demand-side response to new energy integration}

The basic idea of the demand-side response to the large-scale new energy grid-connected role is to influence the controllable load (schedulable demand-side resources) through various demand-side response measures, make it a virtual generator set, and assist the traditional water-fired power generation unit to work together. Adjust the load balance so as to make full use of hydropower and 
distributed energy, promote the large-scale grid-connected renewable energy, and at the same time promote the optimal allocation of inter-provincial interregional large-scale resources, and ultimately achieve energy structure optimization and energy conservation and emission reduction. The effect of demand side response on the integration of renewable energy is mainly reflected in changing the nature of the local load. The Analysis of demand-side response to new energy incorporation is shown in Fig. 1.

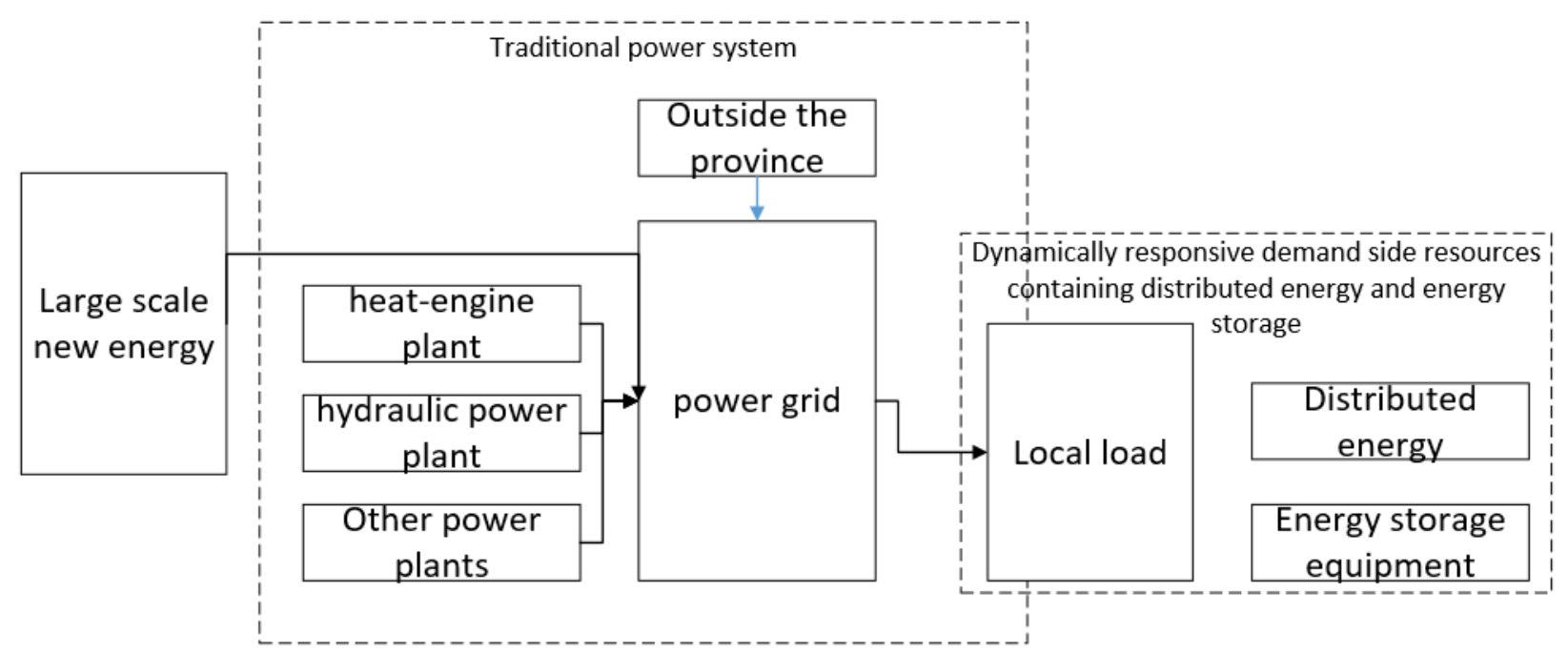

Fig 1. Analysis of demand-side response to new energy incorporation

Turning Traditional Rigid Loads into Flexible Loads. Because the demand-side response can guide the user to change the way of using electricity through price and incentives, so that the user can actively adjust the load, so that the traditional rigid load can be transformed into a flexible load. On the one hand, the load has elasticity, and can be increased or decreased. Characteristics; On the other hand, the load can be transferred with characteristics that can be transferred over time.

Improve Power System Reliability. After the introduction of renewable energy grid-connected on the demand side, the grid dispatching agency can comprehensively utilize the resources on both sides of supply and demand, and can also effectively cope with the impact of renewable energy output fluctuations on the safe and reliable operation of the grid, and thus improve the reliability of the power system .

Improve power system economics. In the power system, the power supply needs to be balanced at all times. If the fluctuation of the power load on the demand side is too large, the renewable energy with poor controllability cannot be connected to the grid on a large scale, and at the same time, a large amount of power generation output is bound to increase. Very low standby power, wasting a lot of human and financial resources. The effective introduction of demand-side response can reduce the construction of standby power supply on the supply side, increase the utilization hours of traditional units, greatly improve the efficiency of the use of renewable energy in grid connection, effectively reduce the operating costs of the supply side system, and further improve the economic efficiency of the power system.

Demand Side Response Increases Distributed Energy Utilization. After the demand-side response measures are implemented, we can first predict the peak period of new energy output according to the distributed new energy generation forecast, and simulate the distribution curve of distributed energy, clearly dividing the peaks and valleys of distributed energy generation. In addition, through the different means of dynamic response on the demand side, users are motivated to actively participate in power demand side management. In the peak period of new energy output, the use of price reductions encourages the increase in electricity consumption, which in turn allows the user to adjust part of the power load to distributed. The peak period of energy generation, thereby increasing the utilization of distributed energy generation. On the contrary, in the low stage of new energy output, 
the overall electricity load is reduced by increasing prices, thereby reducing the traditional thermal power generation, and effectively promoting low-carbon economy and sustainable development.

\section{Demand-side response strategies to promote new energy grid integration}

The selection of demand side response implementation mode is closely related to the degree of power marketization. Under different degrees of marketization, the implementation phase of demand-side response of large-scale grid-connected wind power projects can be divided into the primary market stage, the transitional stage model, and the market improvement stage. Due to the different application conditions of each demand-side response implementation method, The overall implementation phase of the demand side response is shown in Fig. 1.
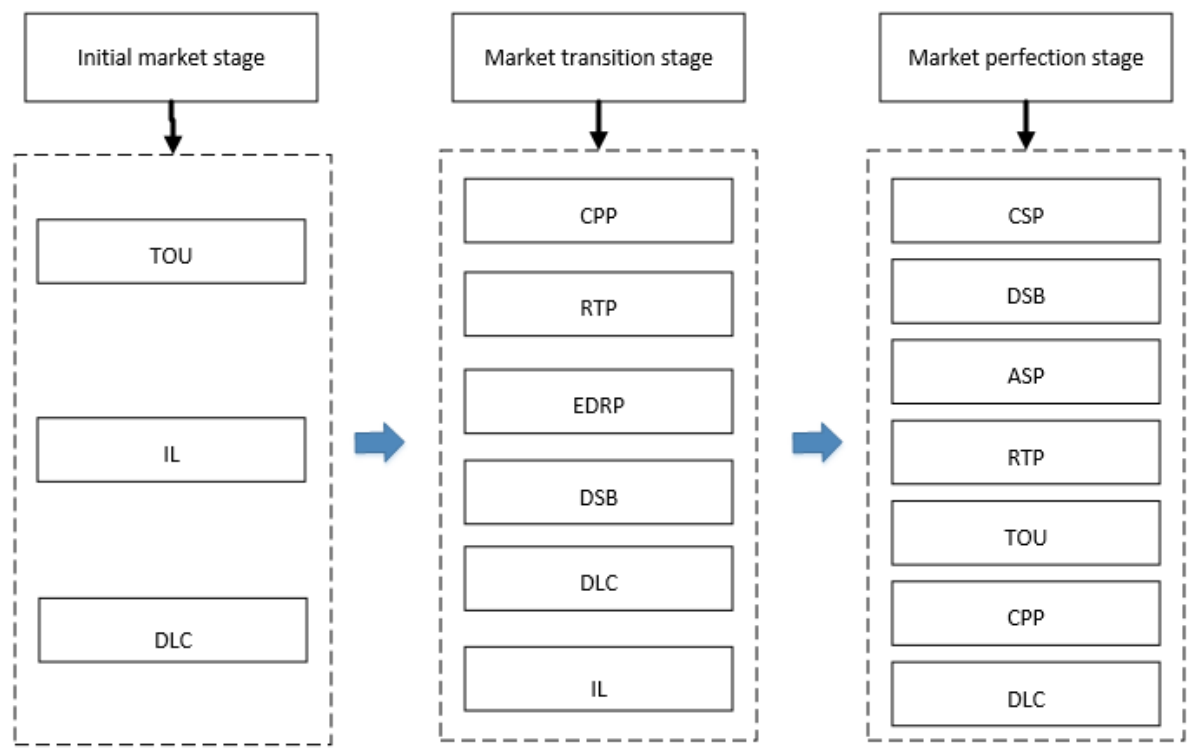

Fig 1. The overall implementation phase of the demand side response

The initial stage of the market. In the initial stage of the market, China's electricity market has not yet formed a complete set of market operation rules and electricity price formation mechanism. It is unable to adjust market supply and demand in an efficient and timely manner to meet the needs of wind power grid integration. Therefore, the implementation mode of the demand-side response at this stage should be based on orderly power management, and the time-of-use price mechanism should be introduced. As shown below.

Market Transition. In the transitional phase of the market, China's electricity market operation rules have been gradually improved, and the electricity price formation mechanism has been initially established. Therefore, it is possible to consider the introduction of corresponding economic measures to increase the response of market players to fluctuations in wind power output. In this stage, the demand-side response implementation mode should be based on the primary stage of the market, and the implementation methods such as TOU, DLC, and IL should be improved, and the key peak charge price should be introduced. (CPP), Real-time Electricity Price (RTP), Emergency Load Response (EDRP), Demand Side Bidding (DSB).

I Timeshare price. In terms of daily load shaping, real-time electricity prices are more efficient. Therefore, in the transitional phase of the market, the time-of-use price mechanism will be improved. On the one hand, the daily peak-to-peak electricity price will be gradually adjusted to the current real-time electricity price. On the other hand, the seasonal hour-by-hour electricity price will continue to be retained.

I Key charge price. Based on the time-based electricity price as a basic load shaping method, a key peak charge price (CPP) is introduced for the special case of sudden drop in wind power output. When the wind power output plummets, the power grid operation and management agency starts 
the key peak charging price and reduces the power load level. The key peak charge price is divided into two types: fixed term (CPPF) and change duration (CPPV). In the fixed-term key peak charge price, the key peak load duration is determined in advance (generally informed one day in advance); the time and duration of the implementation of the key peak charge price during the change period are not determined in advance, usually requires intelligent response, two-way communication And other technical support. In the market transition period, due to the incompleteness of relevant intelligent communication technologies, it is still difficult to implement the key peak charging price of the change period, and the fixed-term key peak charging price can be given priority.

I Real-time electricity prices. Real-time electricity price is an advanced form of peak-to-peak electricity price, and it is more efficient in terms of load shaping. Therefore, in the transition period of the market, the introduction of a real-time electricity price mechanism will help improve the demand-side response effect and ease the adverse effects of fluctuations in wind power output. According to different division methods, there are several types of real-time electricity pricing mechanisms, including those of the previous and the future; mandatory and voluntary.

I Direct load control and interruptable load. In the market transition phase, demand-side direct load control and interruptible load capacity resources for wind power interconnection are further increased, and the scale of direct load control and interruptible load is expanded.

I Emergency load response. Unlike the interruptable load, the load reduction of the emergency load response is voluntary, and the user can ignore the load reduction request without being punished. Therefore, the implementation of emergency load response is more flexible and the response reliability is lower. In the phase of market transition, the introduction of emergency load response on the basis of interruptible load projects will help expand the scale of load reduction projects and acquire more demand-side response resources. .

I Demand side bidding. Introducing bids on the demand side will help increase the enthusiasm of large industrial and commercial users to participate in the demand side response of wind power, and further expand the scale of demand side response projects for wind power integration. Demand-side bids have two implementation modes: In the first mode, the user submits a combination price that includes the price and the amount of load reduction. If the user wins the bid, the next day it must reduce the corresponding load as required, otherwise it must accept one. Penalty; In the second mode, the user as a "price receiver" does not need to submit a bid. It cuts the load according to the requirements of the system operating agency and pays it at the prevailing market price. In the market transition phase, due to the fact that the user's business decision-making technology is not yet mature, it may be considered to first introduce demand-side bids in the form of "price receivers".

Market Improvement Stage. In the mature stage of the market, China's electricity market system has been relatively complete, and various grid operation technologies have become more mature. Therefore, consider introducing more flexible market instruments. As a result, the three major markets, Capacity Market (CSP), Demand Side Bidding Market (DSB) and Auxiliary Services Market (ASP), were formed to improve real-time electricity price (RTP), peak-to-peak price of electricity (TOU) and key peaks. The charge price (CPP) is a demand side response system that uses a direct load control (DLC) as a means of adjustment.

I Price means. Cancel the time-of-day electricity price at Sun Peak Valley and continue to maintain the seasonal hourly electricity price of "heating period-non-heating period"; further expand the proportion of real-time electricity price user groups for wind power integration, and based on wind power forecasting technology and grid intelligence operation control technology, Implementation of the real-time electricity price in the hourly calculation; implementation of the key change time limit price (CPPV).

I Capacity market. Capacity market (CSP) projects can be viewed as a combination of Interruptible Load (IL) projects and Emergency Load Response (EDRP) projects, and are large-scale 
organized implementations of interruptible load projects and emergency load response projects. In this kind of project, the user promises to perform a pre-agreed or specified load reduction in the event of a sharp drop in wind power output. Regardless of whether a reliability event occurs or not, participants can receive a fixed income that is paid on schedule. In the mature stage of the market, based on gradually expanding the scale of interruptible load and emergency load response, a capacity market project for grid integration of wind power is gradually established to provide a stable and reliable response resource for rapid on-grid connection of wind power.

I Demand side bidding market. On the basis of improving the technical decision-making level of large-scale industrial and commercial users, the "price-receiver" implementation model will be gradually transformed into a "competitive bidding" model, and the proportion of large-scale industrial and commercial users participating in demand-side bidding will be continuously increased to finally establish a demand-side bidding market.

I Auxiliary service market. In the ancillary services market, users directly participate in the ancillary services market by using their curtailable load as operating backup. If its bid is accepted, its possible load reduction will be used as a backup, and at the same time, it will receive a same capacity market price payment as the power generation side; after it is actually reducing the load according to the dispatching institution's instructions, it will be charged again according to the spot market power price. Get paid. In the auxiliary service market, participants are generally medium-sized and above users, and the response time is usually measured in minutes. Therefore, more advanced real-time measurement technologies are required to support them. In the mature stage of the market, with the introduction of advanced real-time metrology technology, an auxiliary service market for medium-sized and above users can be established for the integration of wind power.

I Direct load control. The capacity market, the demand-side bidding market and the auxiliary service market are all based on market-oriented measures to guide users to reduce the load according to fluctuations in wind power output. Although the establishment and joint operation of the capacity market, the demand-side bidding market and the auxiliary service market can provide relatively stable demand response for wind power grid connection under normal circumstances, but the market measures lack initiative and reliability and cannot be fast under extreme conditions. Responsive, so in the mature stage of the market, it is still necessary to retain direct load control measures and further increase the direct load control resources for wind power grid connection.

\section{Demand-side response implementation method to promote new energy grid integration}

At present, China's electricity market is still in its infancy. During the "Thirteenth Five-Year Plan" period, with the acceleration of the marketization process in China, the electricity market will also enter the transitional stage. Therefore, we plan the implementation path of China's demand-side response to promote large-scale grid-connected consumption of renewable energy at different stages of the power market.

Resident users. In the initial stage of the market, the time-of-use price is implemented. At this stage, the interval between peaks, sections, and valleys can be set to a relatively large value. At this time, the time-of-use electricity price is relatively low. Simultaneous implementation of seasonal time-sharing electricity prices. At the same time as the implementation, the user is required to conduct a research on the resource potential of the demand side in order to know the user's elasticity of demand in each period, and to provide a basis for further detailed time division.

In the market transition phase, the TOU pricing mechanism in the primary stage is improved. On the one hand, the electricity price period is refined according to the demand-side potential survey; on the other hand, the daily peak-to-hour electricity price is gradually adjusted to the current real-time electricity price; Seasonal time-based electricity prices are still retained. At the same time, pilot work 
was carried out to establish a real-time tariff user group for wind power integration, and to conduct more detailed division of electricity prices.

In the stage of market perfection, based on mature wind power forecasting technology and grid intelligence operation control technology, the real-time electricity price policy in hours is calculated.

Industrial users.In the initial stage of the market, the electricity price policy is still not perfect, and the production equipment control technology is not yet mature. At the same time, due to the adjustability of the production period of industrial users, priority should be given to direct load control and interruptible load control. Electricity prices are secondary. At the same time, the user is required to conduct a research on the resource potential of the demand side in order to know the user's elasticity of demand in each time period, and to provide a basis for further detailed time division.

In the market transition phase, industrial users' control of their electricity demand is gradually strengthened. When the control technology reaches a more flexible level, the key peak charging price can be introduced. Gradually change the daily peak-to-peak electricity price to the current real-time electricity price. At this time, direct load control and interruptible load control should be used as little as possible. At the same time, it gradually transformed the "price receiver" implementation model into a "competitive bidding" model, continuously increased the proportion of users participating in demand-side bidding, and gradually established a capacity market project for wind power integration to provide large-scale grid-connected wind power. Quick response stable and reliable response resources.

In the market improvement stage, the real-time electricity price policy based on hours is fully implemented, and users fully participate in the bidding and capacity market on the demand side. At this time, direct load control measures should still be maintained to deal with the extreme problems that the market cannot solve.

Business users.In the initial stage of the market, due to the inadmissibility of business users' business hours, it is not easy to implement mandatory load control. At the same time, since the energy storage equipment is not perfect, time-of-day electricity price measures that are more time-interval may be adopted. In addition, the research on the resource potential on the demand side provides a basis for further detailed time division.

In the market transition stage, with the large-scale application of energy storage and air conditioning, based on the elasticity of the price of resources on the user's demand side, the time-of-use price period is subdivided, and the real-time electricity price is introduced. Encourage users to participate in the capacity market, demand-side bidding market and ancillary services market.

In the stage of market improvement, the real-time pricing policy based on hours is fully realized. At the same time, based on the maturity and improvement of user business decision-making technology, a complete capacity market, demand-side bidding market and ancillary services market mechanisms have been formed.

\section{Conclusions}

This paper starts with the role of demand response to the new energy grid-connected consumption, combines the characteristics of new energy generation and the development of the power market, and proposes a three-phase demand response strategy, including the primary market stage, market transition stage, and market improvement stage. Finally, in conjunction with the market players, the implementation of demand response measures to promote the integration of new energy sources into the market is proposed. On the one hand, it is beneficial for grid companies to select different demand response resources according to different stages of the market, so that the operation of the grid and new energy consumption can be guaranteed. On the other hand, it is conducive to the implementation of demand response strategies under various market entities to ensure grid companies Can get response in time. 


\section{References}

[1] Xue Song,TU Junming,Yang Su,Zhang Xiaoyi.Resource-side resources in China promote the large-scale intermittent renewable energy grid-connected consumption model[J].Technology Economics and Management Research,2015(07):82-86.

[2] National Development and Reform Commission. Medium and long-term development plan for renewable energy [R]. 2007.

[3]Liu Wei. Analysis of Consumption Strategies under Large-scale Integration of New Energy[J]. Low Carbon World, 2017(34): 17-18.

[4] Liu G. Research on household electricity load control strategy based on demand response [D]. Harbin University of Science and Technology, 2014.

[5] He Yuyu. Research on Multi-user Assistance Wind Power Consumption Optimization Model Based on Demand Response[D].North China Electric Power University(Beijing),2016. 